\title{
STRONG AND UNIFORM CONTINUITY - THE UNIFORM SPACE CASE
}

\author{
DOUGLAS BRIDGES AND LUMINIŢA VÎŢĂ
}

\begin{abstract}
It is proved, within the constructive theory of apartness spaces, that a strongly continuous mapping from a totally bounded uniform space with a countable base of entourages to a uniform space is uniformly continuous. This lifts a result of Ishihara and Schuster from metric to uniform apartness spaces. The paper is part of a systematic development of computable topology using apartness as the fundamental notion.
\end{abstract}

To every uniform structure $\mathcal{U}$ on a set $X$ there corresponds a relation $\bowtie$ of apartness between subsets $S$ and $T$ of $X$, defined by

$$
S \bowtie T \Longleftrightarrow \exists U \in \mathcal{U}(S \times T \subset \sim U) .
$$

In turn, this provides us with a notion of strong continuity for a function $f: X \longrightarrow Y$ between uniform spaces, defined by

$$
\forall S, T \subset X(f(S) \bowtie f(T) \Longrightarrow S \bowtie T) .
$$

It is easy to show that a uniformly continuous map is strongly continuous. Our aim in this paper is to produce a proof of the following partial converse.

THEOREM 1. Let X be a totally bounded uniform space with a countable base of entourages. Then every strongly continuous mapping from X into a uniform space is uniformly continuous.

Our proof is entirely constructive in the sense of Errett Bishop: given a strongly continuous map $f: X \longrightarrow Y$ between uniform spaces, and an entourage $V$ of $Y$, we show (at least in principle) how to find an entourage $U$ of $X$ such that

$$
\forall x, y \in X((x, y) \in U \Longrightarrow(f(x), f(y)) \in V) .
$$

To do this, instead of working with classical logic and a clearly specified notion of algorithm, such as a recursive one, we work with intuitionistic logic and an appropriate informal set theory. It follows that all our results and proofs can easily be translated into any formalism for computable mathematics, such as recursion theory [1, 14] and Weihrauch's type II effectivity theory [19]. They also hold in Brouwer's intuitionistic mathematics [17].

Our paper can be regarded as a contribution to computable topology based on a notion of apartness between subsets of the ambient space. In the general theory [8], strongly continuous mappings are important as the morphisms in the category of sets with apartness. Our result shows that in the subcategory of totally bounded uniform spaces with a countable 
base of entourages, these maps coincide with the usual morphisms. For related work see $[18,8,12,16]$.

Classically, every uniform space with a countable base of entourages is pseudometrisable (see [5, p. 142, Proposition 2]). It is not known whether (and it seems extremely unlikely that) this theorem, with its highly nonconstructive proof, holds constructively. Ishihara and Schuster have shown constructively that every strongly continuous mapping from a totally bounded metric space to a metric space is uniformly continuous [13]. Even if the foregoing pseudometrisation theorem turns out to be constructive, the Ishihara-Schuster result is extended by our Theorem 1 , since the latter does not require that the codomain of the strongly continuous function be metrisable.

To read this paper you do not need any deep understanding of constructive mathematics (mathematics with intuitionistic logic - see $[2,3,4,6,17])$, but some intuitive feel for what is, and what is not, constructive will help. Nor do you need much background knowledge about uniform spaces. However, for the sake of clarity we now present some fundamental definitions in the theory of sets and uniform spaces.

By an inequality on a set $X$, we mean a binary relation $\neq$ that satisfies the following conditions:

$$
\begin{aligned}
& x \neq y \quad \Longrightarrow \quad y \neq x ; \\
& x \neq y \quad \Longrightarrow \quad \neg(x=y) .
\end{aligned}
$$

Note that this notion of inequality is more general than the common classical one (denial of equality).

In the presence of an inequality, we define the complement of a subset $S$ of $X$ to be

$$
\sim S=\{x \in X: \forall s \in S(x \neq s)\} .
$$

Let $X$ be a nonempty set, and let $U$ and $V$ be subsets of the Cartesian product $X \times X$. We define certain associated subsets as follows:

$$
\begin{aligned}
U^{1} & =U, \\
U^{-1} & =\{(x, y):(y, x) \in U\}, \\
U^{n+1} & =U \circ U^{n} \quad(n=1,2, \ldots), \\
U \circ V & =\{(x, y): \exists z \in X((x, z) \in U \wedge(z, y) \in V)\} .
\end{aligned}
$$

We say that $U$ is symmetric if $U=U^{-1}$. The diagonal of $X \times X$ is the set

$$
\Delta=\{(x, x): x \in X\}
$$

A family $U$ of subsets of $X \times X$ is called a uniform structure, or uniformity, on $X$ if the following conditions hold. (Note that condition U1, and the fact that - by U2 - each element of $\mathcal{U}$ is nonempty, show that $\mathcal{U}$ is a filter on $X \times X$.)

U1 (i) Every finite intersection of sets in $u$ belongs to $u$.

(ii) Every subset of $X \times X$ that contains a member of $\mathcal{U}$ is in $\mathcal{U}$.

U2 Every member of $u$ contains both the diagonal $\Delta$ and a symmetric member of $u$.

U3 For each $U \in \mathcal{U}$ there exists $V \in \mathcal{U}$ such that $V^{2} \subset U$.

U4 For each $U \in \mathcal{U}$ there exists $V \in \mathcal{U}$ such that

$$
\forall \mathbf{x} \in X \times X(\mathbf{x} \in U \vee \mathbf{x} \notin V)
$$


The elements of $U$ are called the entourages of (the uniform structure on) $X$. A subfamily $\mathcal{B}$ of $\mathcal{U}$ is called a base of entourages if each element of $U$ contains an element of $\mathcal{B}$.

Metric spaces and locally convex linear spaces are uniform spaces according to this definition.

The uniform topology on a uniform space $(X, \mathcal{U})$ is the topology $\tau_{u}$ in which for each $x \in X$, the sets

$$
U[x]=\{y \in X:(x, y) \in U\} \quad(U \in \mathcal{U})
$$

form a base of neighbourhoods of $x$.

We define the canonical inequality on a uniform space $(X, \mathcal{U})$ by

$$
x \neq y \Longleftrightarrow \exists U \in \mathcal{U}((x, y) \notin U) .
$$

Note that, by axioms U1(ii) and U2, if $U \in \mathcal{U}$, then $U^{-1} \in \mathcal{U}$. It follows that if $x \neq y$, then $y \neq x$. Moreover, since $U$ contains $\Delta$, if $x \neq y$, then $\neg(x=y)$. Thus $\neq$ is indeed an inequality relation on $X$. In turn, we define an associated inequality on $X \times X$ in the obvious way:

$$
(x, y) \neq\left(x^{\prime}, y^{\prime}\right) \Longleftrightarrow\left(x \neq x^{\prime} \vee y \neq y^{\prime}\right) .
$$

It then follows from the axioms that for each $U \in \mathcal{U}$ there exists $V \in \mathcal{U}$ such that $V^{2} \subset U$ and

$$
\forall \mathbf{x} \in X \times X(\mathbf{x} \in U \vee \mathbf{x} \in \sim V) .
$$

Even for the metric apartness space $\mathbf{R}$, the statements ' $x \neq y$ ' and ' $\neg(x=y)$ ' are not constructively equivalent unless we accept the following principle of unbounded search, known as Markov's principle.

For each binary sequence $\left(a_{n}\right)_{n=1}^{\infty}$, if it is impossible that $a_{n}=0$ for all $n$, then there exists $n$ such that $a_{n}=1$.

See [6, Chapter 1].

A mapping $f$ of a uniform space $(X, \mathcal{U})$ into a uniform space $(Y, \mathcal{V})$ is said to be uniformly continuous if

$$
\forall V \in \mathcal{V} \exists U \in \mathcal{U} \forall x, x^{\prime} \in X\left(\left(x, x^{\prime}\right) \in U \Longrightarrow\left(f(x), f\left(x^{\prime}\right)\right) \in V\right) .
$$

A uniform space $(X, \mathcal{U})$ is totally bounded if, for each $U \in U$, there exists a finitely enumerable subset $\left\{x_{1}, \ldots, x_{n}\right\}$ of $X$ such that $X=\bigcup_{k=1}^{n} U\left[x_{k}\right]$. (A set $S$ is finitely enumerable if, for some natural number $n$, there exists a mapping of the set of positive integers less than or equal to $n$ onto $S$.)

LEMma 2. A totally bounded uniform space with a countable base of entourages is separable.

Proof. Let $\left(U_{n}\right)_{n=1}^{\infty}$ be a countable base of entourages for a totally bounded uniform space $(X, U)$. For each $n$ there exists a finitely enumerable subset $F_{n}$ of $X$ such that $X=\bigcup_{x \in F_{n}} U_{n}[x]$. Let $C$ be the countable set $\bigcup_{n=1}^{\infty} F_{n}$. Given $x \in X$ and $U \in U$, find $n$ such that $U_{n} \subset U$. Then there exists $y \in F_{n} \subset C$ such that $(x, y) \in U_{n} \subset U$, so $y \in U[x]$. It follows by the definition of the topology on $X$ that $C$ is dense in $X$.

For convenience below, we define, for each positive integer $n$, an $n$-chain of entourages of $X$ to be an $n$-tuple $\left(U_{1}, \ldots, U_{n}\right)$ of entourages such that for each $k$ (where $1<k \leqslant n$, 
we have $U_{k}$ symmetric, $U_{k}^{2} \subset U_{k-1}$, and

$$
\forall \mathbf{x} \in X \times X\left(\mathbf{x} \in U_{k-1} \vee \mathbf{x} \in \sim U_{k}\right) .
$$

Axiom $\mathrm{U} 3$ ensures that for each $U \in \mathcal{U}$ and each positive integer $n$ there exists an $n$-chain $\left(U_{1}, \ldots, U_{n}\right)$ of entourages with $U_{1}=U$.

The following lemma is crucial to the development of our argument, and is proved in [9, Lemma 1]. It is designed to take the sting out of a number of the succeeding proofs, and is necessitated by the constructive failure of what Bishop called the limited principle of omniscience (LPO):

for each binary sequence $\left(\lambda_{n}\right)_{n=1}^{\infty}$ either $\lambda_{n}=0$ for all $n$, or else there exists $n$ such that $\lambda_{n}=1$.

In its recursive interpretation, the limited principle of omniscience entails the decidability of the halting problem [6, Chapter 3].

Lemma 3. Let $X$ and $Y$ be uniform spaces, let $f: X \longrightarrow Y$ be a strongly continuous function, and let $V$ be an entourage of $Y$. Let $\left(\lambda_{n}\right)_{n=1}^{\infty}$ be an nondecreasing binary sequence, and let $\left(A_{n}\right)_{n=1}^{\infty}$ and $\left(S_{n}\right)_{n=1}^{\infty}$ be sequences of subsets of $X$ such that:

- for each entourage $U$ of $X$ there exists $v$ such that for each $n \geqslant v$, either $A_{n} \times S_{n}=\emptyset$ or else $A_{n} \times S_{n}$ intersects $U$;

- if $\lambda_{n}=0$, then $A_{n}=\emptyset$; and

- if $\lambda_{n}=1-\lambda_{n-1}$, then $A_{n} \neq \emptyset, S_{n} \neq \emptyset, f\left(A_{n}\right) \times f\left(S_{n}\right) \subset \sim V$, and $A_{j}=\emptyset$ for all $j>n$.

Then there exists $N$ such that $\lambda_{n}=\lambda_{N}$ for all $n \geqslant N$.

Two sequences $\left(x_{n}\right)_{n=1}^{\infty}$ and $\left(x_{n}^{\prime}\right)_{n=1}^{\infty}$ in a uniform space $(X, \mathcal{U})$ are said to be eventually close if

$$
\forall U \in U \exists N \forall n \geqslant N\left(\left(x_{n}, x_{n}^{\prime}\right) \in U\right) .
$$

A mapping $f$ of $X$ into a uniform space $Y$ is uniformly sequentially continuous if the sequences $\left(f\left(x_{n}\right)\right)_{n=1}^{\infty}$ and $\left(f\left(x_{n}^{\prime}\right)\right)_{n=1}^{\infty}$ are eventually close in $Y$ whenever $\left(x_{n}\right)_{n=1}^{\infty}$ and $\left(x_{n}^{\prime}\right)_{n=1}^{\infty}$ are eventually close in $X$.

A major step towards our main result is the following weak converse to the proposition that uniform continuity implies strong continuity.

Proposition 4 (see [9, Proposition 6]). A strongly continuous mapping $f: X \longrightarrow Y$ between uniform spaces is uniformly sequentially continuous.

We now establish a number of technical lemmas needed for the proof of our main result, Theorem 1.

LEMMA 5. Let $X$ be a totally bounded uniform space with a countable base of entourages, and $f$ a strongly continuous mapping of $X$ into a uniform space $Y$. Let $\left(x_{n}\right)_{n=1}^{\infty}$ be a dense sequence in $X$ (which exists by Lemma 2), and $V$ any entourage of $Y$. Let $\left(\lambda_{n}\right)_{n=1}^{\infty}$ be an nondecreasing binary sequence, and let $\left(A_{n}\right)_{n=1}^{\infty}$ and $\left(B_{n}\right)_{n=1}^{\infty}$ be sequences of subsets of $X$, such that:

- if $\lambda_{n}=0$, then $A_{n}=\emptyset$, and

- if $\lambda_{n}=1-\lambda_{n-1}$, then $A_{n} \neq \emptyset,\left\{x_{1}, \ldots, x_{n-1}\right\} \subset B_{n}, f\left(A_{n}\right) \times f\left(B_{n}\right) \subset \sim V$, and $A_{j}=\emptyset$ for all $j>n$.

Then there exists $N$ such that $\lambda_{n}=\lambda_{N}$ for all $n \geqslant N$. 
Proof. Take $S_{n}=\left\{x_{1}, \ldots, x_{n-1}\right\}$. Given an entourage $U$ of $X$, compute $N$ such that $X=\bigcup_{i=1}^{N-1} U\left[x_{i}\right]$, and consider any $n \geqslant N$. If either $\lambda_{n}=0$ or $\lambda_{n-1}=1$, then $A_{n} \times S_{n}=\emptyset$. If $\lambda_{n}=1-\lambda_{n-1}$, then $A_{n} \neq \emptyset$ and for each $x \in A_{n}$ there exists $i$ such that $1 \leqslant i \leqslant$ $N-1<n$ and $x \in U\left[x_{i}\right]$; so $A_{n} \times S_{n}$ intersects $U$. Hence we can apply Lemma 3 to obtain the desired result.

LEMMA 6. If $f: X \longrightarrow Y$ is a strongly continuous mapping between uniform spaces, then $f(\bar{S}) \subset \overline{f(S)}$ for each $S \subset X$.

Proof. Let $y=f(x)$, where $x \in \bar{S}$, and let $V$ be any entourage of $Y$. It is enough to show that $f(S)$ intersects $V[y]$. To this end, construct a 3-chain $\left(V_{1}, V_{2}, V_{3}\right)$ of entourages of $Y$ with $V_{1}=V$. We see from [16, Corollary 16 and Lemma 17] that

$$
y \in V_{3}[y] \subset-\sim V_{2}[y] \subset V_{1}[y] .
$$

In particular, $\{y\} \bowtie \sim V_{2}[y]$, so, by the strong continuity of $f$,

$$
\{x\} \bowtie f^{-1}\left(\sim V_{2}[y]\right) .
$$

Choose a 2-chain $\left(U_{1}, U_{2}\right)$ of entourages of $X$ such that

$$
\{x\} \times f^{-1}\left(\sim V_{2}[y]\right) \subset \sim U_{1} .
$$

By [16, Lemmas 13 and 15], we have $U_{2}[x] \subset-f^{-1}\left(\sim V_{2}[y]\right)$. Since $U_{2}[x]$ is a neighbourhood of $x$ in $X$, it follows that there exists $s$ in $S \cap-f^{-1}\left(\sim V_{2}[y]\right)$. Then $f(s) \notin \sim V_{2}[y]$, from which it follows that $f(s) \in V[y]$.

LEMMA 7. Let $X$ be a totally bounded uniform space with a countable base of entourages. Let $f$ be a strongly continuous mapping of $X$ into a uniform space $Y$, let $\left(V_{1}, \ldots, V_{5}\right)$ be a 5-chain of entourages of $X$, and let $S$ be a finitely enumerable subset of $X$. Then:

- either for each $x \in X$ there exists $s \in S$ such that $(f(x), f(s)) \in V_{1}$; or else

- there exists $x \in X$ such that $\{f(x)\} \times f(S) \subset \sim V_{5}$.

Proof. Write $S=\left\{s_{1}, \ldots, s_{M}\right\}$. By Lemma 2, $X$ is separable. Choosing a dense sequence $\left(x_{n}\right)_{n=1}^{\infty}$ in $X$, construct an nondecreasing binary sequence $\left(\lambda_{n}\right)_{n=1}^{\infty}$ such that for each $n$,

$$
\begin{aligned}
\lambda_{n}=0 & \Longrightarrow \forall k \leqslant n \exists i \leqslant M\left(\left(f\left(x_{k}\right), f\left(s_{i}\right)\right) \in V_{4}\right) ; \\
\lambda_{n}=1-\lambda_{n-1} & \Longrightarrow \forall i \leqslant M\left(\left(f\left(x_{n}\right), f\left(s_{i}\right)\right) \in \sim V_{5}\right) .
\end{aligned}
$$

We may assume that $\lambda_{1}=0$. If $\lambda_{n}=0$, set $B_{n}=\emptyset$; if $\lambda_{n}=1-\lambda_{n-1}$, set $B_{n}=$ $\left\{x_{1}, \ldots, x_{n-1}\right\}$ and $B_{k}=\emptyset$ for all $k>n$. Let $B=\bigcup_{n=1}^{\infty} B_{n}$.

Next, construct an nondecreasing binary sequence $\left(\mu_{n}\right)_{n=1}^{\infty}$ such that, for each $n$,

$$
\begin{aligned}
\mu_{n}=0 & \Longrightarrow \forall k \leqslant n \exists i \leqslant M\left(\left(f\left(x_{k}\right), f\left(s_{i}\right)\right) \in V_{2}\right) ; \\
\mu_{n}=1-\mu_{n-1} & \Longrightarrow \forall i \leqslant M\left(\left(f\left(x_{n}\right), f\left(s_{i}\right)\right) \in \sim V_{3}\right) .
\end{aligned}
$$

We may assume that $\mu_{1}=0$. If $\mu_{n}=0$, set $A_{n}=\emptyset$; if $\mu_{n}=1-\mu_{n-1}$, set $A_{n}=\left\{x_{n}\right\}$ and $A_{k}=\emptyset$ for all $k>n$. Let $A=\bigcup_{n=1}^{\infty} A_{n}$. We show that

$$
f(A) \times f(B) \subset \sim V_{5} .
$$

To this end, let $x \in A$ and $y \in B$, and suppose that $(f(x), f(y)) \in V_{4}$. Choosing $n$ such that $y \in B_{n}$, we see that $\lambda_{n}=1-\lambda_{n-1}, B=B_{n}=\left\{x_{1}, \ldots, x_{n-1}\right\}, y=x_{j}$ for some $j \leqslant n-1$, and there exists $i \leqslant M$ such that $\left(f(y), f\left(s_{i}\right)\right) \in V_{4}$. Hence

$$
\left(f(x), f\left(s_{i}\right)\right) \in V_{4}^{2} \subset V_{3} .
$$


On the other hand, since $x \in A$, there exists $k$ such that $\mu_{k}=1-\mu_{k-1}, x=x_{k}$, and $\left(f(x), f\left(s_{i}\right)\right) \in \sim V_{3}$, which contradicts inclusion (2). Hence $\neg\left((f(x), f(y)) \in V_{4}\right)$, and therefore $(f(x), f(y)) \in \sim V_{5}$. Since $x$ and $y$ are arbitrary elements of $A$ and $B$ respectively, we now obtain inclusion (1).

It follows from this and the strong continuity of $f$ that there exists an entourage $U$ of $X$ such that $A \times B \subset \sim U$. Since $X$ is totally bounded, there exists $N$ such that $X=\bigcup_{n=1}^{N} U\left[x_{n}\right]$. If $\lambda_{N}=1$, then there exists $j \leqslant N$ such that $\left\{f\left(x_{j}\right)\right\} \times f(S) \subset \sim V_{5}$. So, without loss of generality, we assume that $\lambda_{N}=0$. Suppose that $\mu_{n}=1-\mu_{n-1}$ for some $n$. Then for each $i \leqslant M$, we have $\left(f\left(x_{n}\right), f\left(s_{i}\right)\right) \in \sim V_{3}$, and therefore $\left(f\left(x_{n}\right), f\left(s_{i}\right)\right) \notin V_{4}$. Hence $\lambda_{m} \neq 0$, and therefore $\lambda_{m}=1-\lambda_{m-1}$, for some $m \leqslant n$; clearly, $N<m$. We now have $A=\left\{x_{n}\right\}, B=\left\{x_{1}, \ldots, x_{m-1}\right\}$, and

$$
\left\{x_{n}\right\} \times\left\{x_{1}, \ldots, x_{m-1}\right\} \subset \sim U,
$$

which is absurd since, by our choice of $N$, there exists $j \leqslant N<m$ such that $\left(x_{n}, x_{j}\right) \in U$. It follows, for all $n$, that $\mu_{n}=0$, and therefore there exists an $i \leqslant M$ such that we have $\left(f\left(x_{n}\right), f\left(s_{i}\right)\right) \in V_{2}$.

Finally, consider any $x \in X$. Since $x$ is in the closure of the set $\left\{x_{n}: n \geqslant 1\right\}$, we see from Lemma 6 that $f(x)$ is in the closure of $\left\{f\left(x_{n}\right): n \geqslant 1\right\}$; whence there exists $n$ such that $f\left(x_{n}\right) \in V_{2}[f(x)]$. Choosing $i \leqslant M$ such that $\left(f\left(x_{n}\right), f\left(s_{i}\right)\right) \in V_{2}$, we have $\left(f(x), f\left(s_{i}\right)\right) \in V_{2}^{2} \subset V_{1}$.

The following proposition, the last link in the chain connecting us to the proof of Theorem 1 , is of interest in its own right and also has an important corollary.

Proposition 8. Let $X$ and $Y$ be uniform apartness spaces, and $f$ a strongly continuous mapping of $X$ into $Y$ such that $f(X)$ is totally bounded. Then $f$ is uniformly continuous.

Proof. Given an entourage $V$ of $Y$, construct a 5-chain $\left(V_{1}, V_{2}, V_{3}, V_{4}, V_{5}\right)$ of entourages of $Y$ such that $V_{2}^{3} \subset V_{1}=V$, and $V_{4}^{3} \subset V_{3}$. Choose $x_{1}, \ldots, x_{m}$ in $X$ such that $Y=$ $Y_{1} \cup \cdots \cup Y_{m}$, where $Y_{i}=V_{4}\left[f\left(x_{i}\right)\right]$; then set $X_{i}=f^{-1}\left(Y_{i}\right)$. For $1 \leqslant i, j \leqslant m$ construct $c_{i j}$ such that

$$
\begin{aligned}
& c_{i j}=0 \Longrightarrow\left(f\left(x_{i}\right), f\left(x_{j}\right)\right) \in V_{2} ; \\
& c_{i j}=1 \Longrightarrow\left(f\left(x_{i}\right), f\left(x_{j}\right)\right) \in \sim V_{3} .
\end{aligned}
$$

For each $(i, j)$ with $c_{i j}=1$, we have $Y_{i} \bowtie Y_{j}$. To see this, consider such $i, j$ and an element $\left(y, y^{\prime}\right)$ of $Y_{i} \times Y_{j}$, and suppose that $\left(y, y^{\prime}\right) \in V_{4}$. Then $\left(f\left(x_{i}\right), y\right) \in V_{4}$ and $\left(y^{\prime}, f\left(x_{j}\right)\right) \in V_{4}$, so $\left(f\left(x_{i}\right), f\left(x_{j}\right)\right) \in V_{4}^{3} \subset V_{3}$, a contradiction. Hence $\left(y, y^{\prime}\right) \in \sim V_{5}$. It follows that $Y_{i} \times Y_{j} \subset \sim V_{5}$, and therefore that $Y_{i} \bowtie Y_{j}$. By the strong continuity of $f$, $X_{i} \bowtie X_{j}$, and therefore there exists an entourage $E_{i j}$ of $X$ such that $X_{i} \times X_{j} \subset \sim E_{i j}$. Let

$$
E=\bigcap\left\{E_{i j}: c_{i j}=1\right\}
$$

which also is an entourage of $X$. Consider points $x$ and $y$ of $X$ with $(x, y) \in E$. Choose $i$ and $j$ such that $f(x) \in Y_{i}$ and $f(y) \in Y_{j}$. If $c_{i j}=1$, then

$$
(x, y) \in X_{i} \times X_{j} \subset \sim E_{i j} \subset \sim E,
$$

a contradiction.

Hence $c_{i j}=0$, and so $\left(f\left(x_{i}\right), f\left(x_{j}\right)\right) \in V_{2}$. Since $\left(f(x), f\left(x_{i}\right)\right) \in V_{4} \subset V_{2}$ and $\left(f(y), f\left(x_{j}\right)\right) \in V_{4} \subset V_{2}$, it follows that $(f(x), f(y)) \in V_{2}^{3}$ and therefore that $(f(x), f(y)) \in V$. 
For those readers prepared to explore the general constructive theory of apartness spaces, we digress briefly to introduce a corollary of Proposition 8.

Let $X$ be any apartness space - that is, a set equipped with a binary relation $\bowtie$ between subsets, for which the axioms in [10] hold. We say that a uniformity $\mathcal{U}$ on $X$ is compatible with the given apartness $\bowtie$ if

$$
S \bowtie T \Longleftrightarrow \exists U \in \mathcal{U}(S \times T \subset \sim U) .
$$

COROLlARY 9. A given apartness space has at most one compatible uniformity that is totally bounded.

Proof. Let $(X, \bowtie)$ be an apartness space, and suppose that there are two totally bounded uniformities $U$ and $U^{\prime}$ that are compatible with the apartness on $X$. Denote the apartness relations corresponding to $U$ and $U^{\prime}$ by $\bowtie u$ and $\bowtie \mathcal{U}^{\prime}$ respectively. Then the identity mapping from $(X, \bowtie)$ onto $(X, \bowtie u)$ is strongly continuous, as is its inverse; likewise, the identity mapping from $(X, \bowtie)$ onto $\left(X, \bowtie u^{\prime}\right)$ is strongly continuous, as is its inverse. Hence the identity mapping from $(X, \bowtie u)$ to $\left(X, \bowtie \mathcal{U}^{\prime}\right)$ is strongly continuous, as is its inverse. It follows from Proposition 8 that the uniformities $\mathcal{U}$ and $\mathcal{U}^{\prime}$ are equivalent.

At last we are in a position to give the proof of Theorem 1.

Proof. Let $X$ be a totally bounded uniform space with a countable base $\left(U_{n}\right)_{n=1}^{\infty}$ of entourages. We may assume that $U_{1} \supset U_{2} \supset \cdots$. Let $\left(x_{k}\right)_{k=1}^{\infty}$ be a dense sequence in $X$. Then there exists a strictly increasing sequence $\left(k_{n}\right)_{n=1}^{\infty}$ of positive integers such that

$$
X=\bigcup_{j=1}^{k_{n}} U_{n}\left[x_{j}\right]
$$

for each $n$. Let $F_{n}=\left\{x_{1}, \ldots, x_{k_{n}}\right\}$. Consider a strongly continuous mapping $f$ of $X$ into a uniform space $Y$. In view of Proposition 8, it is enough to prove that $f(X)$ is totally bounded. Accordingly, given an entourage $V$ of $Y$, construct a 5-chain $\left(V_{1}, \ldots, V_{5}\right)$ of entourages of $Y$ with $V_{1}=V$. Using Lemma 7, construct a nondecreasing binary sequence $\left(\lambda_{n}\right)_{n=1}^{\infty}$ such that

$$
\begin{aligned}
\lambda_{n}=0 & \Longrightarrow \exists x \in X \forall k \leqslant k_{n}\left(\left(f(x), f\left(x_{k}\right)\right) \in \sim V_{5}\right) ; \\
\lambda_{n}=1-\lambda_{n-1} & \Longrightarrow \forall x \in X \exists k \leqslant k_{n}\left(\left(f(x), f\left(x_{k}\right)\right) \in V_{1}\right) .
\end{aligned}
$$

We may assume that $\lambda_{1}=0$. If $\lambda_{n}=0$, set $A_{n}=B_{n}=\emptyset$. If $\lambda_{n}=1-\lambda_{n-1}$, then, as $\lambda_{n-1}=0$, there exists $\zeta \in X$ such that $\left(f(\zeta), f\left(x_{k}\right)\right) \in \sim V_{5}$ for all $k \leqslant k_{n-1}$; set $A_{n}=\{\zeta\}, B_{n}=F_{n-1}$, and $A_{j}=B_{j}=\emptyset$ for all $j \geqslant n$; then $f\left(A_{n}\right) \times f\left(B_{n}\right) \subset \sim V_{5}$. We can now apply Lemma 5 to find $N$ such that if $\lambda_{n}=\lambda_{N}$ for all $n \geqslant N$.

Assume that $\lambda_{N}=0$. Then for each $n, \lambda_{n}=0$ and so there exists $z_{n} \in X$ such that

$$
f\left(z_{n}\right) \times f\left(F_{n}\right) \subset \sim V_{5} .
$$

On the other hand, for each $n$ there exists $\zeta_{n} \in F_{n}$ such that $\left(z_{n}, \zeta_{n}\right) \in U_{n}$. It follows that the sequences $\left(z_{n}\right)_{n=1}^{\infty}$ and $\left(\zeta_{n}\right)_{n=1}^{\infty}$ are eventually close: for if $U$ is any entourage of $X$ and we choose $m$ such that $U_{m} \subset U$, then we have $\left(z_{n}, \zeta_{n}\right) \in U_{n} \subset U_{m} \subset U$ for all $n \geqslant m$. By Proposition 4 , the sequences $\left(f\left(z_{n}\right)\right)_{n=1}^{\infty}$ and $\left(f\left(\zeta_{n}\right)\right)_{n=1}^{\infty}$ are eventually close, so there exists $n$ such that $\left(f\left(z_{n}\right), f\left(\zeta_{n}\right)\right) \in V_{5}$. However, since $\zeta_{n} \in F_{n}$, this contradicts (3). Thus $\lambda_{N} \neq 0$, so $\lambda_{N}=1$ and therefore $f(X)=\bigcup_{j=1}^{k_{N}} V\left[x_{j}\right]$. Since $V$ is an arbitrary entourage of $Y$, we conclude that $f(X)$ is totally bounded. 
Acknowledgement. The authors thank the Marsden Foundation of the Royal Society of New Zealand for supporting Luminiţa Vîţă as a Postdoctoral Research Fellow during the writing of this paper. They also thank Jeremy Clark for his corrections and improvements to the paper.

\section{References}

1. O. Aberth, Computable analysis (McGraw-Hill, New York, 1980). 326

2. M. J. BeEson, Foundations of constructive mathematics, Ergeb. Math. Grenzgeb. (3) 6 (Springer, Berlin, 1985). 327

3. E. A. Bishop, Foundations of constructive analysis (McGraw-Hill, New York, 1967). 327

4. E. A. Bishop and D. S. BRIDGES, Constructive analysis, Grundlehren Math. Wiss. 279 (Springer, Heidelberg, 1985). 327

5. N. BourbaKi, General topology (Springer, Heidelberg, 1989) Chapters 5-10. 327

6. D. S. BRIDGES and F. RichmAN, Varieties of constructive mathematics, London Math. Soc. Lecture Note Ser. 95 (Cambridge Univ. Press, London, 1987). 327, 328, 329

7. D. S. BRIDGES and L. S. VÎŢă, 'Cauchy nets in the constructive theory of apartness spaces', Sci. Math. Jpn. 56 (2002) 123-132.

8. D. S. BRIDGES and L. S. VÎŢĂ, 'Apartness spaces as a framework for constructive topology’, Ann. Pure Appl. Logic 119 (2003) 61-83. 326, 327

9. D. S. BRIDGES and L. S. Vîţă, 'A proof-technique in uniform space theory', J. Symbolic Logic 68 (2003) 95-802. 329

10. D. S. BRIDGES and L.S. VîŢĂ, 'More on Cauchy nets in apartness spaces', Sci. Math. Jpn., to appear. 332

11. D. S. Bridges, H. Ishihara, P. M. Schuster and L. S. Vîţă, 'Strong continuity implies uniform sequential continuity', preprint, University of Canterbury, Christchurch, New Zealand, 2000.

12. D. S. Bridges, P. M. Schuster and L. S. VîŢĂ, 'Apartness, topology, and uniformity: a constructive view', Math. Logic Quarterly 48 (2002) special issue: Computability and complexity in analysis (Proc. Dagstuhl Seminar 01461, 11-16 November 2001) Suppl. 1, 16-28. 327

13. H. IshiHARA and P. M. Schuster, 'A constructive uniform continuity theorem', Quart. J. Math 53 (2002) 185-193. 327

14. B. A. Kushner, Lectures on constructive mathematical analysis, Transl. Math. Monogr. 60 (Amer. Math. Soc., Providence, RI, 1985). 326

15. S. A. Naimpally and B. D. Warrack, Proximity spaces, Cambridge Tracts in Math. and Math. Phys. 59 (Cambridge Univ. Press, London, 1970).

16. P. M. Schuster, L. S. VÎŢă and D. S. Bridges, 'Apartness as a relation between subsets', Combinatorics, computability and logic (Proceedings of DMTCS'01, Constanţa, Romania, 2-6 July 2001), DMTCS Series 17 (ed. C. S. Calude, M. J. Dinneen and S. Sburlan, Springer, London, 2001) 203-214. 327, 330

17. A. S. Troelstra and D. van Dalen, Constructivism in mathematics: an introduction, 2 volumes (North Holland, Amsterdam, 1988). 326, 327 
18. L. S. VÎŢĂ and D. S. BRIDGES, 'A constructive theory of point-set nearness', Theoret. Comput. Sci. 305 (2003) special issue: Topology in computer science: constructivity; asymmetry and partiality; digitization (Proc. Dagstuhl Seminar 00231, 4-9 June 2000, ed. R. Kopperman, M. Smyth and D. Spreen) 473-489. 327

19. K. Weinrauch, Computable analysis, EATCS Texts in Theoret. Comput. Sci. (Springer, Heidelberg, 2000). 326

Douglas Bridges d.bridges@math.canterbury.ac.nz http: / / www . math. canterbury.ac.nz/ mathdsb

Luminiţa Vîţă Luminita@Math. net

http://www.math. canterbury . ac.nz/ mathlsv

Department of Mathematics \& Statistics

University of Canterbury

Private Bag 4800

Christchurch

New Zealand 